\title{
Preparation and Characterization of Keratin-cellulose Composite films
}

\author{
Yuan-Yuan MA ${ }^{\mathrm{a}}$, Ran-Ran $\mathrm{QI}^{\mathrm{b}}$, Shou-Yu $\mathrm{JIA}^{\mathrm{c}}$ and Zhe-Hui WANG ${ }^{*} \mathrm{~d}$ \\ Taishan medical university Tai'an Shadong 710025 China \\ aemail: 1486263693@qq.com, bemail: 1446036692@qq.com, cemail: 756551326@qq.com \\ dwangzh@tsmc.edu.cn \\ *Corresponding author
}

Keywords: Keratin, Cellulose, Composite film, Glutaraldehyde.

\begin{abstract}
In order to improve keratin film mechanical properties, keratin and cellulose solutions were mixed in various ratios and coated on glass plates. Measurements of these composite films show that their mechanical properties decrease with an increase in keratin content in comparison with pure cellulose film. The thermal stability of composite film (40/60) with glutaraldehyde was significantly larger and had a little corrugated surface.
\end{abstract}

\section{Introduction}

Keratin is naturaly abundant as wools, hairs, furs, nails, horns, mammal claws and quills, bird feathers, and the epidermis of reptiles.[1] At the molecular level, the most distinctive feature is the high concentration in cysteine residue (7 20\% of the total amino acid residues).[2] These cysteine residues are oxidized to give inter- and intra- molecular disulfide bonds, which may result in the mechanically strong three-dimensionally linked network of keratin fiber.[3] As a result, this natural polymer is not meltable and not soluble in usual solvents due to its large number of disulfide bonds. And the application and development of keratin is still in its infancy stage. Discarding a large number of keratin not only produces a waste of useful resources, but also pollutes environment. This material presents poor mechanical strength so that the use of cross-linking agents and blending with structural fibrous polymers is often necessary to improve its processing and final mechanical properties.[4] In this paper, we mixed keratin with cellulose to prepare composite films to enhance the mechanical properties. And glutaraldehyde was added into the blended solutions as cross-linking agent.

Various methods used in extraction of keratin from wool are reported in refrences[5 9], and all of them involved the presence of reducing or denaturant agents to break disulfide bonds[4]. At first, 2-mercaptoethanol was usually used as denaturant agent to break disulfide bonds[10], which is highly toxic and has a very pungent odor. Subsequently, some researchers employed sulfides instead of 2-mercaptoethanol[11], which provides an easy route to the wide application of keratin. In this paper, we used $\mathrm{NaHSO}_{3}$ as denaturant agent to break disulfide bonds and SDS as surfactant agent to protect broken disulfide bonds from oxidation by oxygen in the air. Urea solutions of high concentrations were used for breaking large number of hydrogen bonds, ionic bonds and Vander Waals force.

Cellulose as the most abundant organic material resource on earth possesses outstanding properties and extensive applications. It has been estimated that the yearly biomass production of cellulose is 1.5 trillion tons, making it an inexhaustible source of raw material for environment friendly and biocompatible products.[12] But in fact, the efficiency in cellulose utilization is very low, less than $10 \%$. With the exhaustion of oil resources, there has been an enormous interest in making use of cellulose products. Cellulose can be converted in large scale in industry into cellulose derivatives and regenerated materials (fibers, films, food castings, sponges, among others).[13] A major drawback of cellulose material, which limits its utilization, is its low strength, with its wet strength far below the dry one.

The production of regenerated cellulose materials is based largely on more than 100 year old processing technology. However, this process is very complicated and accompanied with environmentally hazardous byproducts $\left(\mathrm{CS}_{2}, \mathrm{H}_{2} \mathrm{~S}\right.$, heavy metals).[13] With technological 
development, a new way has been widely applied in developed countries. The NMMO technology is an environment friendly, resource sustainable and relatively simple method to produce regenerated cellulose materials.[14] In addition, The reagent of NMMO is environment friendly and non-toxic for people, and the percentage of recovery may be as high as $95 \%$ or more. The importance of the NMMO process lies upon the fact that with its strong $\mathrm{N}$-O dipoles, $\mathrm{N}$-methylmorpholine- $\mathrm{N}$-oxide in aqueous solution is capable of dissolving cellulose in a physical way without any derivatization, complexation or special activation.[15]

In this paper, we put keratin into cellulose solution and made keratin-cellulose composite films in order to increase the mechanical property of pure cellulose film and the toughness of pure keratin film. In addition, we have prepared chemical crosslinked keratin-cellulose composite films (40/60) using glutaraldehyde as cross-linking agent and have measured their tensile strength and elongation at break in comparison with keratin-cellulose composite film(40/60). Glutaraldehyde was found to react with the $\alpha$-amino groups of amino acids, the $\mathrm{N}$-terminal amino groups of some peptides and the sulfhydryl group of cysteine. In addition, the phenolic and the imidazole rings of tyrosine and histidine derivatives were partially reactive.[16]

\section{Experimental}

\section{Chemical and Materials}

Wool, cellulose pulp, hexane (Tianjin Yongda Chemical Reagent Co., Tianjin, China), dichloromethane (Hangzhou Gaojing Fine Chemical Industry Co., Hangzhou, China), Urea (Tianjin Yongda Chemical Reagent Co.,Tianjin, China), $\mathrm{NaHSO}_{3}$ (Hangzhou Gaojing Fine Chemical Industry Co., Hangzhou, China), sodium dodecyl sulfate (SDS; Shenergy Biocolor Biotechnology Co., Shanghai, China), NMMO (Aladdin Chemistry Co., Shanghai, China), propyl gallate (Aladdin Chemistry Co., Shanghai, China), formic acid (Shantou Xilong Chemical Factory, Guangdong, China), glutaraldehyde (Sinopharm Chemical Reagent Co., Shanghai, China) were all analytically pure and were used as received.

\section{Preparation of Keratin Solution}

Keratins were extracted according to the method reported previously[5]. But we utilized $\mathrm{NaHSO}_{3}$ instead of 2-mercaptoethano to make the operation more convenient. Briefly, at first , the cleaned wool was soaked in the solvent using a $1: 1 \mathrm{v} / \mathrm{v}$ mixture of hexane and dichloromethane. And then , 10 $\mathrm{g}$ of wool dried was mixed with $8 \mathrm{M}$ urea $(180 \mathrm{ml})$, SDS $(6 \mathrm{~g})$, and $\mathrm{NaHSO}_{3}(10 \mathrm{~g})$ in a $500-\mathrm{mL}$ round-bottom flask and agitated at $90^{\circ} \mathrm{C}$ for $5 \mathrm{~h}$ and then filtered through stainless steel mesh. The filtrate was dialyzed against distilled water (contains a small amount of SDS and $\mathrm{NaHSO}_{3}$ )[17] using cellophane tubing for three days to afford a colorless and clear solution of the reduced keratin. Then the liquid, which was adopted through centrifugation, was cast onto the polypropylene mold and dried at $45^{\circ} \mathrm{C}$ overnight. At last, the pure keratin film was dissolved in the solvent of formic acid[18].

\section{Preparation of Cellulose Solution}

NMMO solution (contains 50\% water) was placed inside the vacuum rotary evaporator IKA RV-10 $\left(0.09 \mathrm{MPa}\right.$ ) at $95^{\circ} \mathrm{C}$ for $2.5 \mathrm{~h}$ in order to reduce its water content to $13.3 \%$ (or below). A $2 \mathrm{wt} \%$ solution of cellulose $(0.5 \mathrm{~g}$ ) in NMMO solution (contains $13.3 \%$ water) was prepared in $100 \mathrm{~mL}$ round-bottom flask and stirred at $110^{\circ} \mathrm{C}$ for $2 \mathrm{~h}$. In order to prevent the degradation of cellulose and NMMO small amount of propyl gallate[19] was added into cellulose-NMMO mixture. Finally, the cellulose solution was transferred into a vacuum oven to remove air bubbles.

\section{Preparation of Keratin-cellulose Films}

Keratin and cellulose solutions were blended at $85^{\circ} \mathrm{C}$ for $2 \mathrm{~h}$ in the ratios of $0 / 100,10 / 90,20 / 80$, 30/70, 40/60 (keratin/cellulose). Then, the blends were coated onto glass plates with square area of about $40 \mathrm{~cm}^{2}(8 * 5 \mathrm{~cm})$ at $95^{\circ} \mathrm{C}$ and soaked in a coagulation bath (distilled water) for 15 minutes, 
which was carried out in a vacuum rotary evaporator in order to recover NMMO. The transparent keratin-cellulose films were washed with plenty of water and dried at room temperature.

\section{Mechanical Properties}

Mechanical properties were measured using a Universal Testing Machine model 5500 at a test speed of $0.5 \mathrm{~cm} / \mathrm{min}$. Samples were $4 \mathrm{~cm}$ by $0.5 \mathrm{~cm}$. And a minimum of five samples of each composite film were tested.

\section{Thermal Analysis}

The thermal stabilities were measured with a Pyris-1 TGA, each sample with an amount around 10 $\mathrm{mg}$ and at a heating rate of $10^{\circ} \mathrm{C} / \mathrm{min}$.

\section{Scanning Electron Microscopy (SEM)}

Microscopic investigation was carried out with a JSM-5610 LV scanning electron microscope (SEM), using an accelerant voltage of $8 \mathrm{kV}$. The fracture surfaces of the films were treated in liquid nitrogen. The films were mounted onto aluminum stub using double-side adhesive tape and sputter-coated with a gold layer at vacuum, using an Emitech K 550 Sputter Coater, with a current of $10 \mathrm{~mA}$ for $40 \mathrm{~s}$.

\section{Fourier Transform Infrared (FT-IR) Spectroscopy}

Keratin-cellulose films were analyzed using a Nicolet 5700 FT-IR in ATR mode. Sixty-four scans at a resolution of $4 \mathrm{~cm}^{-1}$ were used. Background spectra were obtained before each scan and blanks were run between each sample scan to allow for quantitative FT-IR analysis.

\section{Results and Discussion}

\section{FT-IR spectra of Composite Films}

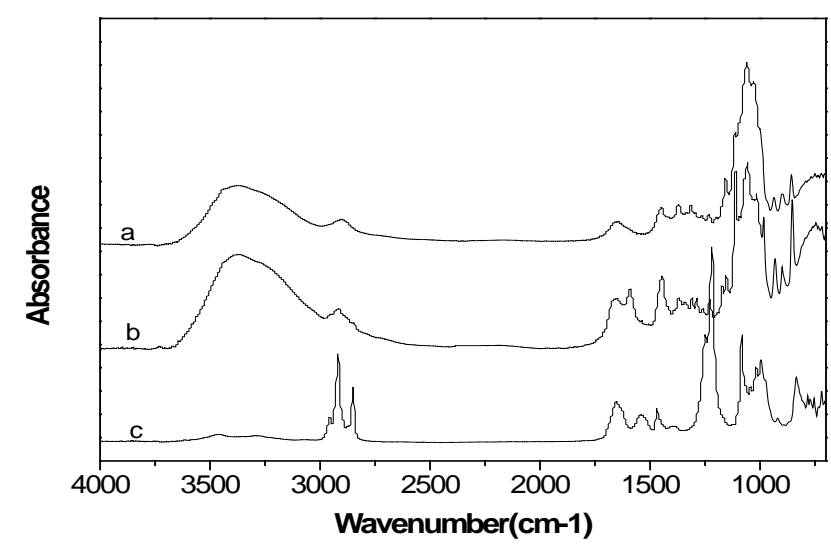

Fig.1. FT-IR spectra of films. (a) pure cellulose (b) KE/CE 20/80composite films (c) pure keratin

The structure changes in keratin-cellulose composite films were studied by comparing FT-IR spectra of the pure cellulose film, the pure keratin film and the keratin-cellulose composite films as shown in Fig. 1. The band at $3367 \mathrm{~cm}^{-1}$ observed in the IR spectrum of pure cellulose film is attributed to the -OH stretching. The peak at $1060 \mathrm{~cm}^{-1}$ is ascribed to the C-O stretching of the polymer backbone. As seen in the spectrum of the pure keratin film, the peaks at $2917 \mathrm{~cm}^{-1}$ and $2850 \mathrm{~cm}^{-1}$ are attributed to the methyl and methylene, respectively, which appear when the disulfide bonds were broken by the denaturant agents. The very intense characteristic band at $1652 \mathrm{~cm}^{-1}$ is due to the $\mathrm{C}=\mathrm{O}$ asymmetric stretching in the carboxylate anion that is reconfirmed by another sharp peak at $1540 \mathrm{~cm}^{-1}$, which is related to the symmetric stretching mode of the carboxylate anion. In addition, the peak at $1082 \mathrm{~cm}^{-1}$ corresponding to the S-O of cysteine monoxide, which was broken disulfide bonds oxidized again[20]. As seen in the IR spectrum of the composite film (20/80), the characteristic peaks of cellulose are still available at $3367 \mathrm{~cm}^{-1}, 1060 \mathrm{~cm}^{-1}$. But a new peak at 1595 is assigned to the 
characteristic absorption bands of the free amino group. Therefore, the $\mathrm{N}-\mathrm{H}$ in the composite film shifted in comparison with one of pure keratin film. As seen in Fig.2, the IR spectrum of composite film (40/60) with glutaraldehyde didn't change significantly in comparison with the spectrum of the composite film (40/60), which may be due to the small content of crosslinker. A new peak at 1455 $\mathrm{cm}^{-1}$ occurred and is attributed to the characteristic absorption bands of the C-O.

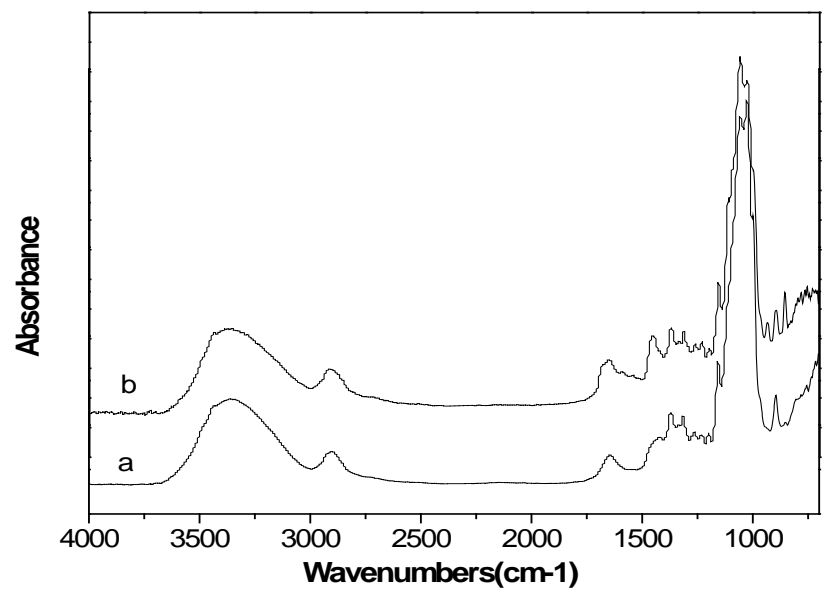

Fig.2. FT-IR spectra of KE/CE blend films (a) without glutaraldehyde (40/60) (b) with glutaraldehyde (40/60)

The mechanical properties of keratin-cellulose composite films are summarized in Fig.3. The pure keratin film is so fragile as not to be measured for its mechanical property, that is the reason that the pure keratin film doesn't have practical value. However, the pure cellulose film was fairly flexible and strong with its ultimate strength (44.3MPa) and ultimate elongation (6.95\%). While the tensile strength of the film prepared from cellulose and $10 \mathrm{wt} \%$ keratin was a little smaller with its ultimate strength (33.4MPa). The addition of keratin made its ultimate strength smaller. Therefore, addition of keratin could decrease the mechanical properties of cellulose film. The reason for this phenomenon may be explained in this way: when keratin dissolves in formic acid, a part of peptide chains are broken because of the strong acidity of formic acid, and the keratin/formic acid solution made the tensile strength of the cellulose film a littler smaller.

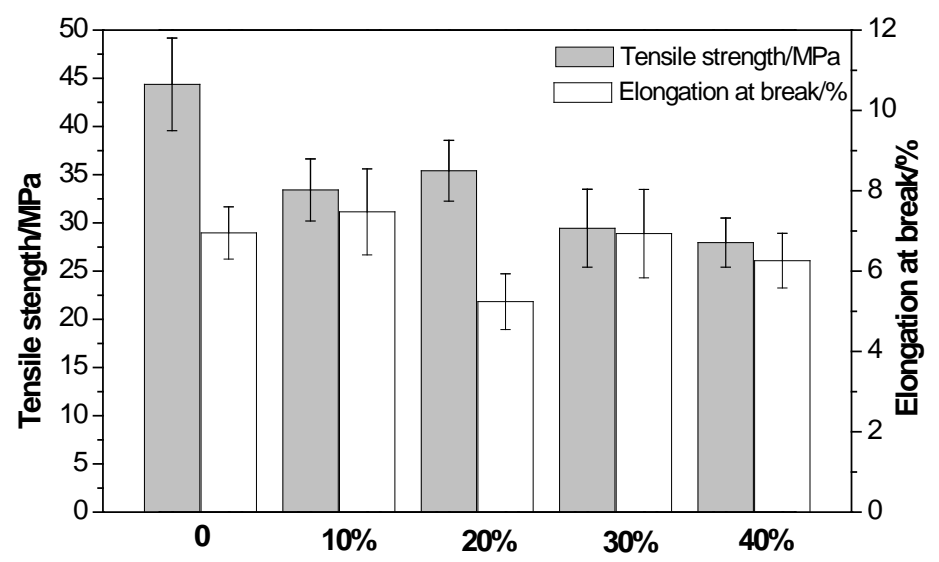

Fig. 3 Mechanical properties of pure CE and KE/CE blends

As shown in Fig.4, the keratin-cellulose film with $40 \mathrm{wt} \%$ keratin is $28.0 \mathrm{MPa}$ in ultimate strength, $6.26 \%$ in ultimate elongation. However, the composite film (40/60) with $0.6 \mathrm{wt} \%$ glutaraldehyde enhanced the ultimate strength (46.6MPa) and ultimate elongation (10.7\%). Glutaraldehyde may react with a large number of $-\mathrm{NH}_{2}$ of keratin and form $\mathrm{C}=\mathrm{N}$. This was the point where the cellulose and keratin were packed together closely and this type of system can have reinforcement effect because of cellulose/keratin interactions. 
As seen in Fig.5, the thermal decomposition of pure CE occurred at $300^{\circ} \mathrm{C}$, and at this stage, the weight loss continued up to $400^{\circ} \mathrm{C}$, and a residue of $29.2 \mathrm{wt} \%$ remained after heating $\mathrm{CE}$ to $410{ }^{\circ} \mathrm{C}$. When keratin was introduced into CE, no matter how little the amount of keratin is, the thermal stabilities decreased slightly. The major decomposition steps of keratin-cellulose films basically focused on $280^{\circ} \mathrm{C}$.

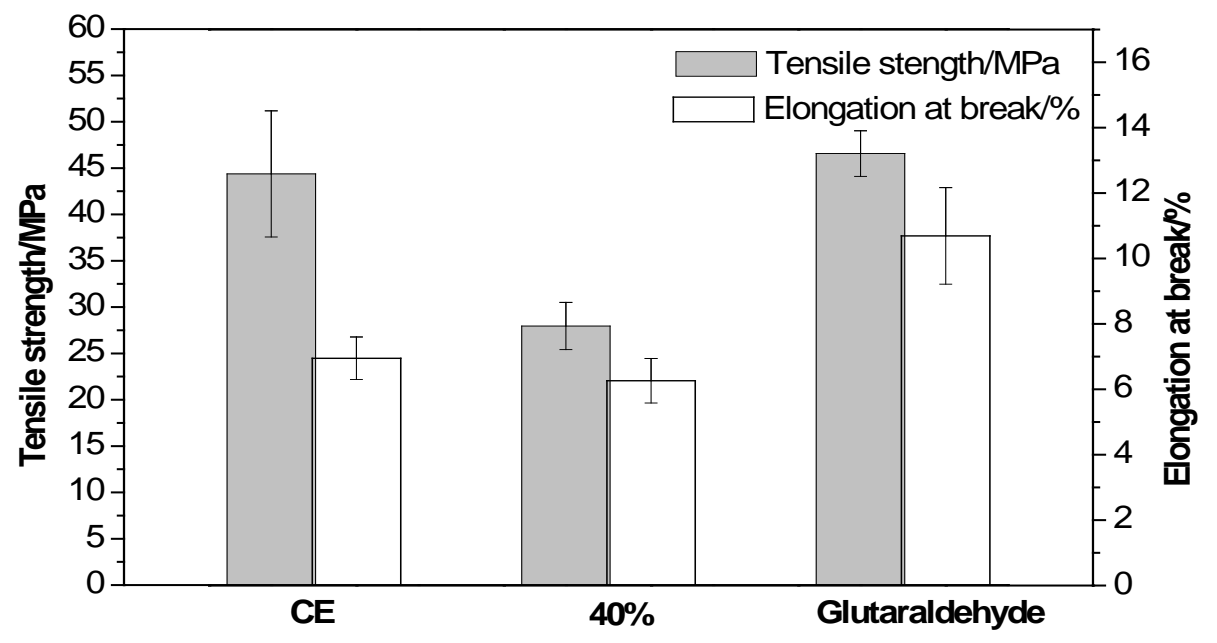

Fig.4 Mechanical properties of pure CE and KE/CE blends

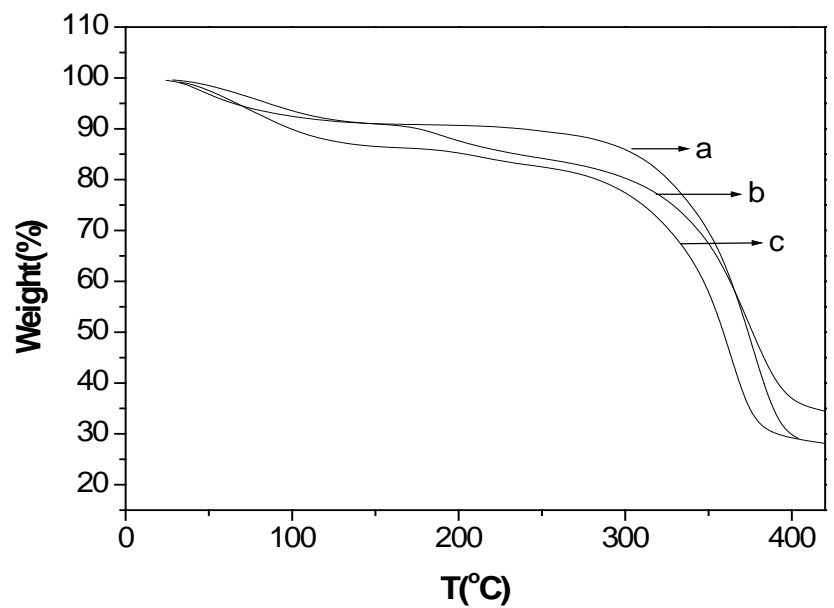

Fig.5 Thermogravimetry of pure KE, CE and KE/CE blends. (a) pure cellulose (b) 30/70 (c) pure keratin

\section{Summary}

The mechanical properties of the composite films declined a little with the rise in keratin content. But the mechanical property of the composite film with glutaraldehyde was increased. In particular, the elongation at break increased by $70 \%$ compared to the composite film (40/60) and increased by 54\% compared to the pure cellulose film. The thermal stability of the composite films without glutaraldehyde decreased a little, but the one with glutaraldehyde increased. So with glutaraldehyde as cross-linking agent, we can get an enhanced mechanical property and thermal stability. The surfaces of composite films become rougher compared with the pure cellulose film, and the surface of composite film with glutaraldehyde become a little corrugated. Consequently, with keratin and cross-linking agent we can get better morphologies of cellulose film. 


\section{References}

[1] M.Zoccola, A.Aluigi, C.Vineis, C.Tonin, F.Ferrero, M. G.Piacentino, Study on cast membranes and electrospun nanofibers made from keratin/fibroin blends, Biomacromolecules 9 (2008) 2819-2825.

[2] L. M.Dowling, W. G.Crewther, D. A. D.Parry, Secondary structure of component 8c-1 of alpha-keratin. An analysis of the amino acid sequence, Biochem J 236(1986) 705-712.

[3] T.Tanabe, N.Okitsu, A.Tachibana, K.Yamauchi, Preparation and characterization of keratin-chitosan composite film, Biomaterials 23(2002) 817-825.

[4] A,Vasconcelos, G.Freddi, C.Artur, Biodegradable materials based on silk fibroin and keratin, Biomacromolecules 9(2008) 1299-1305.

[5] H. J.Rhodes, B.Potter, A.Widra, Characteristics of the alpha-keratose fraction of hair inducing ascosporogenesis in Nannizzia Grubyia, Mycropathol Etmyclogia Appl 33(1967) 345-348.

[6] H.Thomas, A.Conrads, K. H.Phan, L. M.Vande, H.Zahn, In vitro reconstitution of wool intermediate filaments, J. Biol Macromol 8(1986) 258-264.

[7] M.Ito, T.Tazawa, N.Shimizu, A.Saito, Y.Sato, I.Nonaka, Intraepidermal pilar epithelioma: A new dermatopathologic interpretation of a skin tumor, J. American Academy of Dermatology, 86(1988) 123-132.

[8] H.Sakabe, H.Ito, T.Miyamoto, H.Inaga, States of Water Sorbed on Wool as Studied by Differential Scanning Calorimetry, Textile Research Journal, 57 (1987) 66 - 72.

[9] K. Yamauchi , T. Yagi , S. Kuwano, Suppression of the Purgative Action of Rhein Anthrone, the Active Metabolite of Sennosides A and B, by Calcium Channel Blockers, Calmodulin Antagonists and Indometacin ,Pharmacology 62(1993) 22-31,.

[10] K.Yamauchi, A.Yamauchi, T.Kusunoki, A.Kohda, Y.Konishi, Preparation of stable aqueous solution of keratins, and physiochemical and biodegradational properties of films, Journal of Biomedical Materials Res 31(1996) 439-444.

[11] K.Yamauchi, Preparation of collagen/calcium phosphate multilayer sheet using enzymatic mineralization, Biomaterials 25(2004) 5481-5489.

[12] D.Klemm, B.Heublein, H. P.Fink, A.Bohn, Cellulose: fascinating biopolymer and sustainable raw material,Angew. Chem, Int. Ed 44(2005) 3358-3393.

[13] H.Fink, P.Weigel, H. J.Purz, Structure formation of regenerated cellulose materials from NMMO-solutions, J. Prog. Polym. Sci. 26(2001) 1473-1524.

[14] I.Hofmann, D.Haas, A.Eckert, H.Rüf, H.Firgo, F.A.Müller, P.Greil, Mechanical properties of cellulose-apatite composite fibres for biomedical applications, Advances in Applied Ceramics, 107(2008) 293-297.

[15] N.E. Franks, J.K. Varga, US Patent US85495777A (1979).

[16] A.Habeeb, R.Hiramoto, Reaction of proteins with glutaraldehyde, Archives of Biochemistry and Biophysics 126(1968) 16-26.

[17] P. M. M.Schrooyen, P. J.Dijkstra, R. C.Oberthur, A.Bantjes, J. Feijen, Stabilization of Solutions of Feather Keratins by Sodium Dodecyl Sulfate, Journal of Colloid and Interface Science 240(2001) 30-39.

[18] J. R.Barone, W. F.Schmidt, Effect of formic acid exposure on keratin fiber derived from poultry feather biomass, Bioresource Technology 97(2005) 233-242. 
[19] T.Rosenau, A.Potthast, I.Adorjan, A.Hofinger, H.Sixta, H. F. P. Kosma, Cellulose solutions in N -methylmorpholine- N -oxide (NMMO) - degradation processes and stabilizers, Cellulose 9(2002) 283-291.

[20] M. A.Moharram, G. M. S.El-Bahy, S.M.Rabie, Irradiation of wool fibers in mixtures of organic solvents, Polymer - Plastics Technology and Engineering, 36(1997) 635-646. 